\title{
ESTUDO DE VIABILIDADE DE ALOENXERTO DE TRAQUÉIA EM RATOS - O PAPEL DA OMENTOPLASTIA NA VIGÊNCIA DE IMUNOSSUPRESSÃO
}

\author{
STUDY OF RAT'S TRACHEAL ALLOGRAFT VIABILITY: THE ROLE FOR \\ OMENTOPLASTY WITH IMUNOSSUPRESSION
}

\author{
Airton Schneider, TCBC-RS ${ }^{1}$ \\ Tiago Rosito ${ }^{2}$ \\ Vinícios Von Diemen ${ }^{2}$
}

\begin{abstract}
RESUMO: A cirurgia de traquéia enfrenta dificuldades em acompanhar os recentes avanços da terapêutica cirúrgica. Os resultados cirúrgicos são baseados em trabalhos clássicos desenvolvidos há mais de dez anos. Características específicas de vascularização e de regeneração estão entre os problemas que os cirurgiões enfrentam no tratamento das lesões adquiridas ou congênitas da traquéia. As próteses e o transplante ainda não fazem parte do arsenal terapêutico. Com o objetivo específico de determinar o valor da gordura na viabilidade de aloenxertos traqueais, os autores utilizaram ratos Fischer 344 para estudar a regeneração e a viabilidade de aloenxertos, associados à imunossupressão. Um doador possibilitou o implante de um fragmento traqueal em gordura do subcutâneo de dez ratos e de um fragmento no omento. Estes fragmentos de traquéia foram estudados histologicamente visando determinar a viabilidade. Os resultados demonstraram que a gordura extraperitonial, no rato, não serve para manter a viabilidade de aloenxerto, mesmo utilizando imunossupressão $(\mathrm{p}<0,05)$, quando comparada à gordura intraperitoneal.
\end{abstract}

Unitermos: Transplante traqueal experimental, Aloenxerto de traquéia em ratos, Cirurgia traqueal experimental.

\section{INTRODUÇÃO}

Os recentes avanços tecnológicos para o suporte do paciente submetido à cirurgia torácica e a investigação acerca de sua base científica vêm proporcionando expansão da pesquisa básica em cirurgia. Muito se sabe hoje sobre câncer de pulmão, ressecções pulmonares e grande impulso vem sendo dado ao transplante pulmonar. Contudo, ainda há muito a progredir e talvez a traquéia seja, entre todos, o campo mais fértil e que ainda encerra o maior número de questões a serem respondidas.

Grillo ${ }^{1}$, em revisão sobre o assunto, relata que foi nos últimos dez anos que testemunhamos os maiores avanços da cirurgia traqueal: ressecções de lesões extensas, concepção de técnica anestésica adequada e crescente en- tendimento sobre as patologias traqueais. Os trabalhos de Cooper e Grillo ${ }^{2}$ descreveram a estenose traqueal pósentubação, entendida a partir de então como evolução de necrose por isquemia. Tais resultados foram corroborados por Pearson ${ }^{3}$, em estudo clínico retrospectivo. Recentemente, tem-se acompanhado novo alento à investigação traqueal, buscando entender os mecanismos histológicos, reproduzindo estenoses traqueais em laboratório e estudando a revascularização e conseqüente possibilidade de utilizar enxertos traqueais. ${ }^{4-7}$

A imunossupressão tem sido estudada, associada à cirurgia traqueal, com o propósito de estabelecer a viabilidade de aloenxertos traqueais. ${ }^{8-10}$ Também tem-se visto a realização de transplantes "estadiados", mantendo, temporariamente, um segmento traqueal envolto em omento, com posterior relocação à traquéia. ${ }^{8,11,12}$

1. Professor Adjunto do Departamento de Ciências Morfológicas do Instituto de Ciências Básicas da Saúde da UFRGS.

2. Acadêmicos de Medicina da UFRGS.

Recebido em 19/7/99

Aceito para publicação 14/4/2000

Trabalho desenvolvido no Laboratório de Anatomia Experimental, Instituto de Ciência Básicas da Saúde - ICBS, Universidade Federal do Rio Grande do Sul - UFRGS. 
Entretanto, os diferentes estudos utilizam cães como animal experimental, o que no nosso meio é bastante dispendioso, ou coelhos, em um estudo revisado. Os critérios de viabilidade traqueal são confusos e não contemplam consenso na literatura revisada. 4,6,7,11,12

Ainda há muito a responder sobre o reparo de lesões extensas traqueais, por estenose ou não, uma vez que as próteses traqueais ainda encerram grande insucesso terapêutico. ${ }^{11}$

\section{MATERIAL E MÉTODOS}

O estudo foi desenvolvido no Laboratório de Anatomia Experimental da UFRGS, usando ratos machos tipo Fischer 344, randomizados ao acaso, pesando entre 250 e 300 gramas, colocados em gaiolas separadas e tratados conforme normas internacionais de utilização de animais de laboratório. ${ }^{12}$ A amostra foi estimada em 30 ratos, sendo dez para cada grupo de diferente forma de proteção a ser explicada a seguir e dez como animais doadores de segmentos traqueais.

Todos os animais foram anestesiados com pentobarbital sódico na dose de $50 \mathrm{mg} / \mathrm{kg}$ intraperitoneal ${ }^{13}$, sendo aplicada nova dose conforme a necessidade durante os procedimentos. Todos os animais, por receberem alotransplante, foram submetidos à imunossupressão com ciclosporina na dose de $15 \mathrm{mg} / \mathrm{kg} / \mathrm{dia}$ e metilprednisolona na dose de $2 \mathrm{mg} / \mathrm{kg} / \mathrm{dia}$ usados por via subcutânea. ${ }^{8}$

0s animais foram divididos em dois grupos, com os procedimentos a seguir descritos: (todos os segmentos traqueais foram retirados de ratos, que doaram - grupo doador - dez ratos - dois segmentos traqueais de seis anéis cada, sendo eutanasiados com superdosagem de anestésico, logo após a doação - ratos "doadores"). Um fragmento traqueal de cada rato doador foi estudado histologicamente, sendo definido como grupo controle. Os critérios de viabilidade do enxerto foram previamente definidos, tendo como base estudos já realizados ${ }^{4}$, e são descritos a seguir: A) Critério macroscópico: presença ou não de hiperemia na mucosa

B) Critérios microscópicos:

1. descamação focal

2. composição morfológica do epitélio pseudo-estratificado ciliar

3. edema ou dilatação vascular na submucosa

4. presença de cílios na mucosa

Grupo I - receptores 1 - Alotransplante traqueal em subcutâneo: foram utilizados dez animais, anestesiados conforme técnica descrita, recebendo um segmento traqueal no subcutâneo abdominal. Os animais foram mantidos em imunossupressão, conforme já referido, sendo sacrificados em 20 dias ou antes, caso apresentassem sofrimento. Foi retirado o segmento traqueal transplantado para análise histológica, seguindo critérios de viabilidade previamente definidos.

Grupo 2 - receptores 2 - Alotransplante traqueal em omento: foram utilizados dez animais, submetidos à anestesia e imunossupressão, conforme descrito, recebendo segmentos traqueais dos animais "doadores". Cada segmento traqueal de seis anéis foi implantado no grande omento, por meio de laparotomia mediana, com "envelopamento" do enxerto e fixação por meio de fio inabsorvível. Os enxertos foram mantidos no abdome por 20 dias. Após, foram retirados e realizada biópsia de ambas as extremidades para análise histológica. Os segmentos foram estudados histologicamente conforme os parâmetros definidos.

Foi feita a comparação entre os três grupos: controle, enxerto traqueal no subcutâneo e no omento.

Para análise estatística foi utilizado para avaliação de preservação ou não do enxerto (viabilidade) o teste Quiquadrado, sendo definido o nível de significância (alfa) de 0,05 . Para avaliar os diferentes critérios de viabilidade, definidos isoladamente de forma prospectiva, foi utilizada uma análise de variância. Como a distribuição se apresentou de forma normal, foi utilizado o teste de Kruskall-Wallis, sendo determinado um nível de significância (alfa) menor que 0,05 .

\section{RESULTADOS}

Entre os 20 ratos operados, houve quatro perdas em pós-operatório imediato. No grupo de dez ratos de preservação no subcutâneo, um não sobreviveu. Entre os nove ratos, quando comparados com o grupo controle, todos apresentaram anemia da mucosa como critério macroscópico. A textura da traquéia estava amolecida ao toque e à manipulação.

Os achados microscópicos também foram homogêneos. Basicamente, todas as peças estudadas apresentaram ausência ou severa diminuição do número de cílios na mucosa, edema e/ou dilatação da rede vascular da submucosa e destruição do epitélio pseudo-estratificado ciliar. Identificou-se severo infiltrado inflamatório e ausência de glândulas na mucosa (Figura 1).

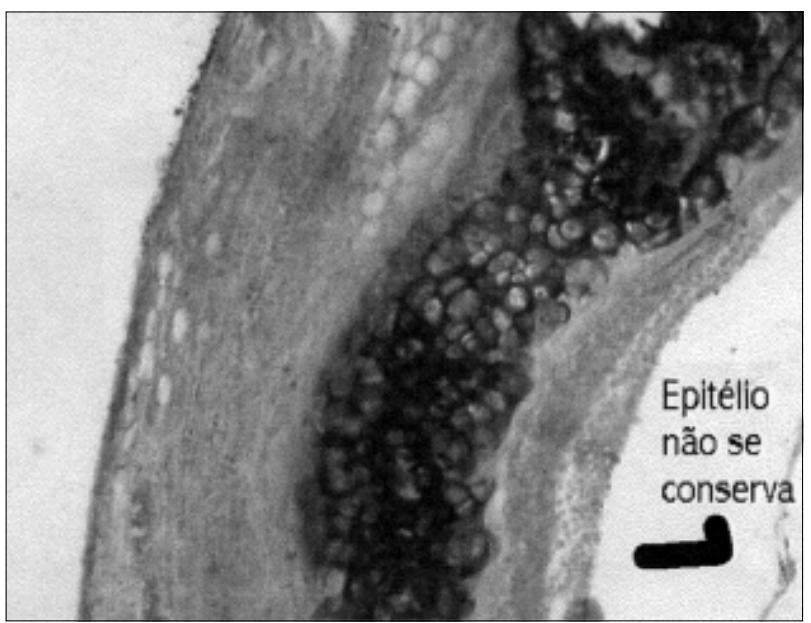

Figura 1 - Traquéia preservada em gordura do subcutâneo: nota-se severa inflamação e não preservação da característica normal do epitélio. 
Entre o grupo com omentoplastia, houve três perdas. Nos sete ratos estudados, a mucosa permaneceu hiperemiada, da mesma cor e textura daquelas que serviram como padrão (Figura 2). Os ratos continuaram a apresentar, quando comparados com o grupo controle, os critérios definidos previamente de viabilidade, como ausência de descamação marcada do epitélio, preservação do epitélio, pouco ou nenhum edema da rede vascular submucosa e presença de cílios. A mucosa permaneceu com textura e coloração normal. Houve leve infiltrado inflamatório e as glândulas da mucosa estavam presentes.

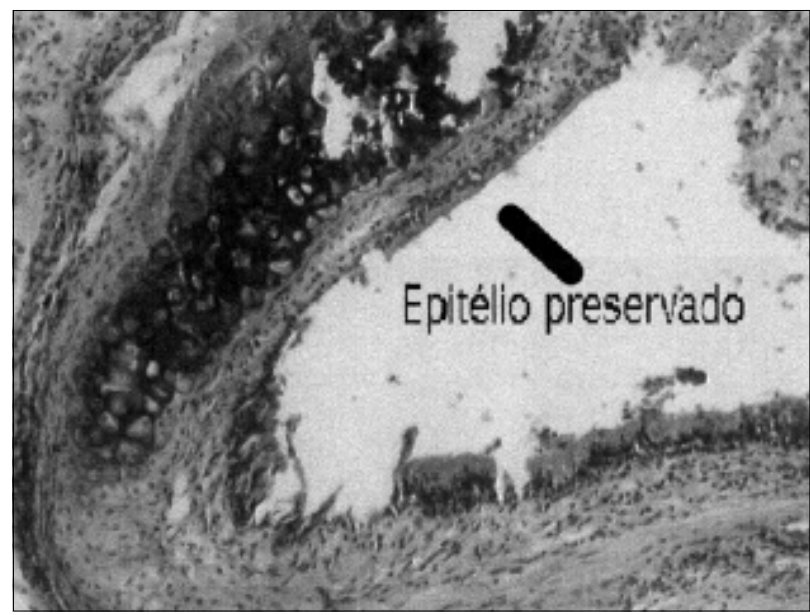

Figura 2 - Traquéia colocada em omento: nota-se uma semelhança com epitélio normal, como razoável preservação da arquitetura.

Quando testados estatisticamente os resultados, na comparação entre a viabilidade do epitélio com as duas técnicas descritas, o omento se mostrou superior à técnica de utilização de gordura subcutâneo $(\mathrm{p}<0,01)$.

Entre os diferentes critérios definidos como de viabilidade, todos apresentaram diferença estatística quando testados isoladamente, conforme está resumido na Tabela 1.

Tabela 1

Comparação de critérios de viabilidade isoladamente

\begin{tabular}{l|c|c|c}
\hline Critério & $\begin{array}{c}\text { Omento } \\
\mathrm{n}=7\end{array}$ & $\begin{array}{c}\text { Subcutâneo } \\
\mathrm{n}=9\end{array}$ & $\mathrm{p}$ \\
\hline $\begin{array}{l}\text { Edema/dilatação } \\
\text { vasc. submucosa }\end{array}$ & - & + & $<0,05$ \\
$\begin{array}{l}\text { Destruição do epitélio } \\
\text { Descamação focal }\end{array}$ & - & + & $<0,05$ \\
Presença de cílios & + & + & $<0,05$ \\
Infiltrado inflamatório & $\begin{array}{c}\text { Nenhum } \\
\text { ou leve }\end{array}$ & Severo & $<0,05$ \\
& & & \\
\hline
\end{tabular}

\section{DISCUSSÃO}

Apesar de amplamente realizadas, as cirurgias traqueais, nos últimos anos, não têm sido investigadas do ponto de vista experimental, principalmente sob a ótica dos mecanismos de regeneração e cicatrização. ${ }^{4}$ Os trabalhos experimentais publicados na literatura por Patterson ${ }^{7,8,10}$ e Lutz Freitag ${ }^{5}$ trouxeram importantes contribuições nesta área: o omento foi utilizado como local de testes, mantendo o enxerto traqueal vascularizado e viável, sem, no entanto, interpor o mesmo na traquéia dos animais. ${ }^{6,9,10,11} \mathrm{O}$ risco aumentado de desvascularização do enxerto quando colocado in situ, sem proteção, desautoriza qualquer estudo clínico de transplante traqueal, devido aos resultados catastróficos obtidos nos estudos experimentais $4,6,7,11,12$.

No presente estudo, além de utilizar animal de baixo custo, propõe-se ampliar as comparações realizadas já descritas na literatura, associando o alotransplante à imunossupressão. Visa-se a definir a importância da rejeição na gênese da desvascularização e da viabilidade.

Desejava-se acompanhar a evolução histológica da regeneração traqueal quando comparado o segmento traqueal transplantado ao omento e quando transplantado em outro sítio com gordura - o subcutâneo, na vigência de imunossupressão.

Visava-se, também, definir de forma clara o papel do omento como receptor produzindo vascularização: se não houvesse diferença estatística em ambos grupos, reforçava-se a idéia de que outros sítios de gordura poderiam ser utilizados para neoangiogênese do enxerto, desde que fosse utilizada imunossupressão. Se por outro lado, comprovada diferença histológica, seria da desvascularização a responsabilidade de maus resultados com cicatrização traqueal. Sendo assim, a neoangiogênese do tecido peritoneal (omento) seria mais eficiente do que gordura extraperitonial (subcutâneo).

Os resultados corroboraram os achados já descritos na literatura, mesmo utilizando imunossupressão. A gordura peritoneal é, pelo menos no rato, capaz de impedir as alterações epiteliais produzidas por isquemia e promove uma proteção estatisticamente superior, quando comparada à gordura subcutânea. A ação de neoangiogênese deve-se, provavelmente, à adição de gordura com células mesoteliais encontradas na cavidade peritoneal. ${ }^{4}$

Os achados deste trabalho sugerem que outros sítios de gordura extraperitonial não servem para proteger e manter a viabilidade de um segmento traqueal. Entretanto, um maior número de animais se faz necessário para extrapolar estas conclusões para a prática clínica. 


\begin{abstract}
The surgery of trachea has so many problems with grafts viability that recent advances did not promote satisfactory results in tracheal replacement surgery. Peculiar aspects of its circulation and cicatrization Larries problems in surgery. Our aim is to show the value of extraperitonial fat to promote tracheal grafts viability of used 20 tracheal segments of rats Fisher 344 that were implanted in the omentum (10) and in the subcutaneous fat (10). The animals were sacrificed after 20 days to evaluate the viability of tracheal grafts. All animals underment imunossupression with ciclosporin and corticosteroids. Tracheal grafts into the omentum were viable in all rats while the protector effects of extraperitonial fat were uneffective $(p<0,05)$. Our conclusion was that tracheal grafts were not be able to viability in the extraperitonial fat.
\end{abstract}

Key words: Tracheal transplantation in rats; Omentoplasty; Experimental tracheal surgery

\title{
REFERÊNCIAS
}

1. Grillo HC. Notes on the windpipe. Ann Thorac Surg 1989; 47:9-26.

2. Cooper JD, Grillo HC. The evolution of tracheal injury due to ventilatory assistance through cuffed tubes. A pathologic study. Ann Surg 1969; 169: 334.

3. Pearson FG, Goldberg M, de Silva AJ. Tracheal stenosis complicating tracheostomy with cuffed tubes: clinical experience and observations from a prospective study. Arch Surg 1968; 97:380.

4. Inayama Y, Tomiyama I, Akaike M et al . Morphologic alteration and cytokinetic studies of tracheal autograft epithelium in rabbits. Ann Thorac Surg 1995; 60: 952-7.

5. Marquette $\mathrm{CH}$, Mensier E, Copin $\mathrm{MC}$ et al. Experimental models of tracheobronchial stenoses: a usefi.tool for evaluating airvay stents. Ann Thorac Surg1995; 60:651-6.

6. Albes JM, Klenzner T. Kotzerke J, Thiedemann KU, SchafersHJ, Borst HG. Improvement of tracheal autogaft revascularization by means of fibroblast growth factor. Ann Thorac Surg 1994; 57:444-9.

7. Olech VM, Keshavjee SH, Chamberlain DW, Slutsky AS, Patterson GA. Role of basic fibroblast growth factor in revascularization of rabbit tracheal autografts. Ann Thorac Surg 1991; 52: 258-64.

8. Davreux CJ, Chu NH . Waddell TK, Mayer E, Patterson A. Improved tracheal allograft viability in imunosuppressed rats. Ann Thorac Surg 1993; 55:131-4.

9. Nakanishi R, Yasumoto K. Minimal dose of cyclosporin A for tracheal allograft. Ann Thorac Surg 1995; 60: 635-9.

10. Smith DE, Waddell TK, DeCampos KN, Slutski AS, Patterson GA. Effect of methilprednisolona on angiogenesis in syngeneic rat tracheal grafts. Ann Thorac Surg 1994; 57:652-6.

11. Li J, Xu P, Chen H, Yang Z, 7hang Q. Improvement of tracheal autograft survivaI with transplantation into the greater omentum. Ann Thorac Surg 1995; 60: 1592-6.

12. Nakarshi R, Shirakusa T, Takachi T. Omentopexy for tracheal autografts. Ann Thorac Surg 1994; 57:841-5.

13. Schneider A. Estudo comparativo de diferentes formas de proteção em modelo de fístula brônquica em ratos. Tese de doutorado, 1995; UFRGS.

\section{ENDEREÇO PARA CORRESPONDÊNCIA}

Dr. Airton Schneider

Instituto de Ciências Básicas da Saúde - UFRGS

Dep. Ciências Morfológicas -

Rua Sarmento Leite 500

90050-170 - Porto Alegre-RS

Telefone/fax: 51-3163146.

E-mail: profairt@vortex.ufrgs.com.br 